\title{
A Model Reduction Algorithm for Solving Multiple Scattering Problems Using Iterative Methods
}

\author{
Alexandre Vion, Ruth V. Sabariego, and Christophe Geuzaine \\ University of Liège, Department of Electrical Engineering and Computer Science, Montefiore Institute, B-4000 Liège, Belgium \\ In this paper we propose a new method for solving high-frequency scattering problems by multiple objects. A model reduction algo- \\ rithm based on the macro basis function (MBF) method is used to find an approximate solution within a subspace spanned by the solutions \\ of several single scattering subproblems. Different iterative methods for generating the MBFs are compared. The whole process relies \\ on a finite element approach and is applied to convex obstacle scattering.
}

Index Terms-Finite-element methods (FEMs), iterative techniques, macro basis function methods, multiple electromagnetic scattering, reduced-order systems, short-wave problem.

\section{INTRODUCTION}

$\mathbf{S}$ OLVING multiple-scattering problems can be a real challenge, especially when the wavelength is significantly smaller than the size of the scattering obstacles.

For non-convex geometries obtained as the union of a finite number of convex surfaces, an efficient algorithm was proposed in [1] based on three main elements: 1) an iteratively computable Neumann series for the currents induced on the scattering surfaces, which rigorously accounts for multiple scattering; 2) a generalized ansatz that allows for a priori determination of the highly oscillatory phase of the currents in each term of the series; and 3) the use of the single-scattering boundary-integral solver from [2] for the efficient evaluation of each term of this series. A finite elements reformulation algorithm was recently proposed in [3], where the steps 2) and 3) described above were adapted to compute the fields in the volume instead of only on the boundary of the scatterers. The iterative process was then accelerated with a phase reduction procedure [4], [5].

In this paper, macro basis functions (MBFs) [6] are introduced to allow for a model-order reduction, which aims at further reducing the number of iterations. The underlying idea is based on the observation that the multiple iterations in the scheme produce subsolutions that span an interesting subspace of the whole solution space, which allows us to solve (a reduced version of) the problem in this subspace.

The paper is organized as follows. First the multiple scattering problem is reformulated in terms of coupled single-scattering subproblems in Section II. Its iterative solution by means of different iterative methods is shown in Section III. Next the MBFs are introduced and linked to the solutions of the single scattering subproblems. The iterative schemes are then modified to further speed up their convergence using the MBF technique. A numerical comparison of the methods on several test cases is finally given.

Manuscript received May 31, 2010; accepted September 07, 2010. Date of current version April 22, 2011. Corresponding author: A. Vion (e-mail: a.vion@ulg.ac.be).

\section{Multiple Scattering AS Coupled Single OBSTACLE SCATTERING}

We study the solution $u(\boldsymbol{x})$ of a scalar time-harmonic scattering problem where the incoming plane wave $u_{\text {inc }}(\boldsymbol{x})$ hits a set of obstacles $\Omega_{s}^{-} \subset \mathbb{R}^{2}, s=1, \ldots, S$, with closed boundaries $\Gamma_{s}$. The real wavenumber $k$ is related to the wavelength $\lambda$ by $\lambda=2 \pi / k$. Let $\Omega^{-}=\cup_{s=1}^{S} \Omega_{s}^{-}$and $\Gamma=\cup_{s=1}^{S} \Gamma_{s}$ be, respectively, the region occupied by the scatterers and its boundary. The original multiple scattering problem in the computational domain $\Omega^{+}=\mathbb{R}^{2} \backslash \overline{\Omega^{-}}$, with Dirichlet boundary conditions on $\Gamma$ and radiation condition at infinity reads

$$
\begin{cases}\Delta u+k^{2} u=0, & \text { in } \Omega^{+} \\ u=-u^{\text {inc }}, & \text { on } \Gamma \\ \lim _{|\boldsymbol{x}| \rightarrow \infty} \sqrt{|\boldsymbol{x}|}\left(\nabla u \cdot \frac{\boldsymbol{x}}{|\boldsymbol{x}|}-i k u\right)=0 . & \end{cases}
$$

The solution $u(\boldsymbol{x})$ can also be written in terms of a sum of $S$ subsolutions

$$
u(\boldsymbol{x})=\sum_{s=1}^{S} u_{s}(\boldsymbol{x}), \quad \boldsymbol{x} \in \Omega^{+}
$$

with $u_{s}$ the solution of a subproblem where the $s$ th scatterer is considered separately in the computational domain $\Omega_{s}^{+}=$ $\mathbb{R}^{2} \backslash \overline{\Omega_{s}^{-}}$(see Fig. 1)

$$
\begin{cases}\Delta u_{s}+k^{2} u_{s}=0, & \text { in } \Omega_{s}^{+} \\ u_{s}=-u^{\text {inc }}-\sum_{r=1, r \neq s}^{S} u_{r}, & \text { on } \Gamma_{s} \\ \lim _{|\boldsymbol{x}| \rightarrow \infty} \sqrt{|\boldsymbol{x}|}\left(\nabla u_{s} \cdot \frac{\boldsymbol{x}}{|\boldsymbol{x}|}-i k u_{s}\right)=0 . & \end{cases}
$$

The subproblems are coupled through the boundary conditions imposed on $\Gamma_{s}$ in (3), where the contributions of all other obstacles are taken into account. It can be shown that the family of $S$ subproblems admits a unique solution [7]. Moreover, when all the scatterers are convex, the use of iterative linear solvers to find an approximate solution of (2), (3) in terms of the single scattering subproblems has been proved to be advantageous [3]. 


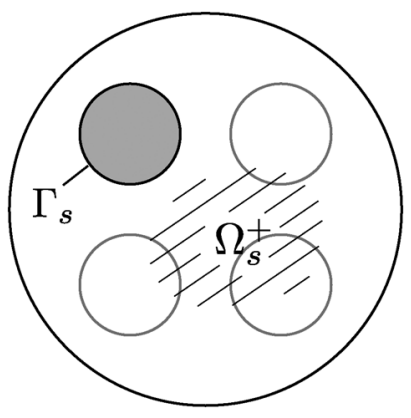

Fig. 1. Computational domain for the $s$ th subproblem.

\section{ITERATIVE SOLUTION TO THE COUPLED SUBPROBLEMS}

Instead of directly solving (3), we look for the solution in terms of the series

$$
u(\boldsymbol{x})=\sum_{m=1}^{\infty} \sum_{s=1}^{S} u_{s}^{(m)}(\boldsymbol{x}), \quad \boldsymbol{x} \in \Omega^{+} .
$$

At each iteration $m$, we solve $S$ scattering problems around the single obstacles $\Omega_{s}^{-}$

$$
\begin{cases}\Delta u_{s}^{(m)}+k^{2} u_{s}^{(m)}=0, & \text { in } \Omega_{s}^{+} \\ u_{s}^{(m)}=c_{s}^{(m)}, & \text { on } \Gamma_{s} \\ \lim _{|\boldsymbol{x}| \rightarrow+\infty} \sqrt{|\boldsymbol{x}|}\left(\nabla u_{s}^{(m)} \cdot \frac{\boldsymbol{x}}{|\boldsymbol{x}|}-i k u_{s}^{(m)}\right)=0 & \end{cases}
$$

with

$$
c_{s}^{(m)}= \begin{cases}-u^{\mathrm{inc}}-\sum_{r=1}^{s-1} u_{r}^{(m)}, & \text { for } m=1 \\ -\sum_{r=1}^{s-1} u_{r}^{(m)}-\sum_{r=s+1}^{S} u_{r}^{(m-1)}, & \text { for } m>1\end{cases}
$$

for a Gauss-Seidel-type iteration. Each correction $u_{s}^{(m)}$ can be interpreted as the correction introduced by the $m$ th wave reflection, the iteration being stopped when the norm of all corrections at step $m$ is smaller than a prescribed tolerance. More sophisticated iterative schemes like Krylov subspace methods can also readily be applied if (3) is reformulated in terms of an iteration operator (see [3]). Furthermore, if the scatterers are convex, a phase reduction procedure can be applied in order to accelerate the solution of each subproblem [3].

\section{Model Reduction Using MBFs}

Let us assume that we have a mesh (e.g., a triangulation) $\Omega_{h}^{+}$ of $\Omega^{+}$. The MBFs, also referred to as characteristic basis functions (CBFs), are a class of basis functions with the entire computational $\Omega_{h}^{+}$as support [8]—in contrast with standard finite element basis functions, the support of which is generally restricted to a few mesh elements (e.g., those touching a given mesh vertex for standard P1 elements). The MBFs are constructed as a linear combination of the standard basis functions, and can thus be seen as higher level basis functions, built on top of the standard ones. As such, if we denote by $W^{0}\left(\Omega_{h}^{+}\right)$the $N$-dimensional finite-element space in which we are seeking the discrete solution of the problem, a reduced set of $P$ linearly independent MBFs spans a $P$-dimensional subspace of solutions $W^{\mathrm{MBF}}\left(\Omega_{h}^{+}\right) \subset W^{0}\left(\Omega_{h}^{+}\right)$. An approximation $\tilde{u}(\boldsymbol{x})$ of the solution to the original problem can of course be expressed in $\Omega_{h}^{+}$ as a linear combination of the $P$ MBFs

$$
\tilde{u}(\boldsymbol{x})=\sum_{k=1}^{P} \alpha_{k} u_{k}(\boldsymbol{x}) .
$$

In matrix form, we can write

$$
\tilde{\mathbf{u}}=P_{\mathrm{MBF}} \boldsymbol{\alpha}
$$

where $P_{\mathrm{MBF}}=\left[\mathbf{u}_{1}, \ldots, \mathbf{u}_{P}\right]$ is the MBF basis of $W^{\mathrm{MBF}}\left(\Omega_{h}^{+}\right)$, and $\boldsymbol{\alpha}=\left[\alpha_{1}, \ldots, \alpha_{P}\right]$ is the vector of coordinates of $\tilde{u}(\boldsymbol{x})$ in that basis.

Although that subspace does not necessarily contain the standard FE solution, its orthogonal projection onto this subspace is its best approximation in the least-square sense. If the discretized problem to solve reads

$$
A \tilde{\mathbf{u}}=\mathbf{b}
$$

and a basis $P_{\mathrm{MBF}}$ of dimension $P$ is available, the coordinates $\boldsymbol{\alpha}$ of the projected solution in (8) are obtained by solving

$$
A_{\text {red }} \boldsymbol{\alpha}=P_{\mathrm{MBF}}^{T} \mathbf{b}
$$

with $A_{\text {red }}=P_{\mathrm{MBF}}^{T} A P_{\mathrm{MBF}}$ the reduced matrix of the new system. Although this $P \times P$ matrix is dense, it should be much smaller than the $N \times N$ original system matrix. Equation (10) can thus be straightforwardly solved to find the approximate solution (without solving the original system).

In a given MBF set, the projected solution is unique and so are the coordinates $\boldsymbol{\alpha}$. The quality of the approximation therefore depends on the choice of the constituent MBFs. A suitable and relevant set of MBFs must be built accounting for the physics of the problem and incorporating all the a priori information. For instance, if the solution is expected to be oscillatory, any constant function will be most likely orthogonal to the solution and is useless in the MBF set.

\section{ITERATIVE MBF GENERATION}

As emphasized in [6], the key point in the model reduction process is the generation of the MBFs. We would like to build as quickly as possible the smallest set of MBFs spanning a subspace in which a sufficiently good approximation of the solution can be found. The coordinates of the projected solution are then easily computed by solving the reduced system (10).

As a starting point, let us consider the solutions of the coupled subproblems defined by the individual obstacles $\left[\mathbf{u}_{1}, \ldots, \mathbf{u}_{S}\right]$ as a MBF set. Obviously, the subspace they span contains the exact solution [compare (2) with (7)]. However, the constraints imposed on these particular basis functions are very strong as they should form a unique set of $S$ MBFs, with all coefficients in $\boldsymbol{\alpha}$ equal to 1. Determining them is costly, as solving the coupled subproblems directly is usually computationally more expensive than solving the original problem. As an alternative, we 
can use the basis formed by the $u_{s}^{(m)}$ iterates in (4). This set of basis functions is less constrained: it depends on the chosen iterative scheme and we are free to truncate the series after any number of terms. If all (an infinity of) $u_{s}^{(m)}$ are considered, their coefficients are once again equal to 1 .

From the above considerations we thus propose to solve the overall problem by modifying the iterative solution of (4) by taking advantage of the intermediate results. We build the MBF set at each iteration with all the "unconverged" subsolutions, i.e., after each iteration cycle, the MBF set is enriched with the current solution:

$$
P_{\mathrm{MBF}}^{(m)}=\left[\left[\mathbf{u}_{1}^{(1)}, \ldots, \mathbf{u}_{S}^{(1)}\right], \ldots,\left[\mathbf{u}_{1}^{(m)}, \ldots, \mathbf{u}_{S}^{(m)}\right]\right]
$$

where $\mathbf{u}_{s}^{(m)}$ is the solution vector to the sth subproblem computed after the $m$ th iteration.

The advantage of this approach is that if the iterative process converges to the solution of the coupled subproblems as defined by (4), the MBF set containing all the intermediate results will eventually contain the solution to the full problem.

Another interesting point is that even if the original iterative scheme fails to converge (as can happen at some particular frequencies for non-Krylov solvers [3], when numerical errors lead to an eigenvalue of the iteration operator becoming slightly larger than 1 [11]), it does not necessarily mean that a good approximation cannot be found in the basis built during the iterative procedure. Indeed, we have observed that the number of iterations required to find a solution within the MBF subspace is still relatively small when the solver eventually fails to converge with the non-MBF approach. Therefore, the introduction of the MBFs in the iterative scheme not only speeds up convergence, but also tends to stabilize it.

In any case, in order to avoid linearly dependent basis functions, an SVD is performed after each iteration to disregard the vectors with the smallest singular values and ensure a good conditioning of the reduced matrix $A_{\text {red }}$. As computing the SVD rapidly becomes costly when the MBF basis grows, one could separate the basis into the vectors associated with each scatterer, so as to compute several SVDs of smaller size - this makes sense since the solutions of different subproblems are linearly independent.

Other choices are possible for the iterative generation of the MBF set. In [12], a Method of Moments approach is presented as well as the link with Krylov subspace solvers.

\section{NUMERICAL RESULTS}

The method has been applied to the scattering of an incident plane wave along the $x$-direction by a collection of cylindrical obstacles in a regular arrangement. Different configurations of the obstacles have been considered, as illustrated on Fig. 2 . A Bayliss-Gunzburger-Turkel-like radiation condition was applied on the outer boundary to truncate the domain [9]. The convergence criterion was set as $\varepsilon=\|A \tilde{\mathbf{u}}-\mathbf{b}\|<1 e^{-4} / k$, with $k$ the wavenumber and $\|\cdot\|$ the 2-norm.

As in [3], three different linear solvers are investigated: Jacobi, Gauss-Seidel, and GMRes [10], [11]. Values of $k R$ ranging from 4 to 25 have been tried and results are reported for

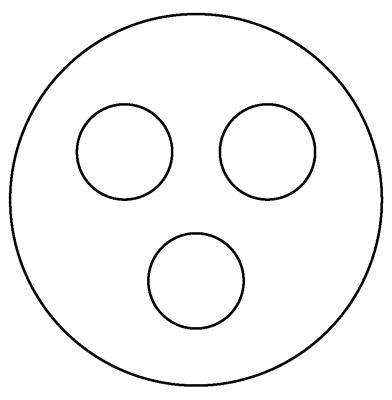

(a)

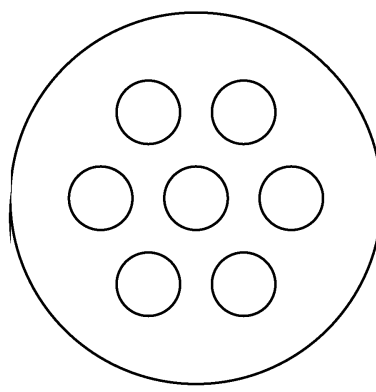

(c)

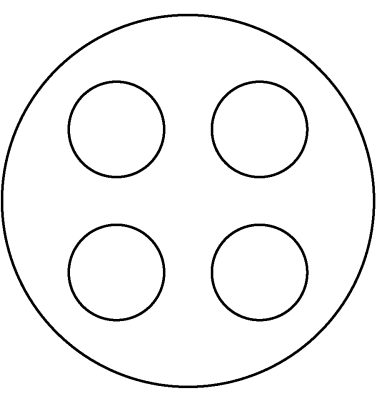

(b)

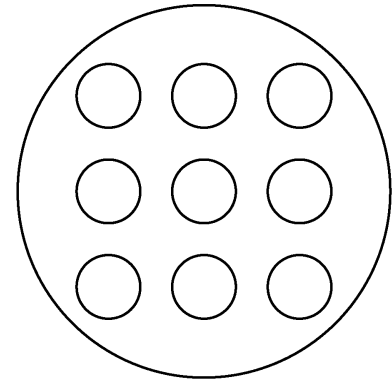

(d)
Fig. 2. Different geometries used. Obstacles with unit radius $R$, separated by a distance $d=R$, taken to 1 in all experiments.

$k R=11$ ( $R$ denotes the radius of the cylindrical scatterers). The behavior was similar at all frequencies.

Fig. 3 shows the convergence rate of the different solvers with respect to the residue criterion, with and without the MBF extension. One clearly observes a reduction in the number of iterations with the new method for all three solvers. Fig. 3(c) and (d) show some interesting cases where both the unmodified Jacobi and Gauss-Seidel solvers fail to converge while the MBF version is still able to converge quickly, highlighting the stabilization of the method provided by the introduction of the MBFs.

Note that the generated subspaces depend on the initial guess $x_{0}$. After $n$ iterations, the GMRes has produced the subspace $x_{0}+\operatorname{span}\left\{r_{0}, \mathcal{A} r_{0}, \mathcal{A}^{2} r_{0}, \ldots, \mathcal{A}^{n-1} r_{0}\right\}$, with $\mathcal{A}$ the iteration operator and $r_{0}=b-\mathcal{A} x_{0}$ [11], that becomes $\operatorname{span}\left\{b, \mathcal{A} b, \mathcal{A}^{2} b, \ldots, \mathcal{A}^{n-1} b\right\}$ in the particular case $x_{0}=0$. Our Jacobi procedure gives raise to exactly this subspace when started with $x_{0}=b$. That is the reason why the convergence of Jacobi + MBF and GMRes + MBF shown on Figs. 3(a), (b), and (d) are almost identical. Fig. 3(c) illustrates the different case where $x_{0}=b$ for both schemes.

In all considered cases, the modified Gauss-Seidel solver requires the least iterations to converge, suggesting that it gives rise to the best MBF set. This can be understood as the GaussSeidel procedure providing an implicit preconditioning of the system.

\section{CONCLUSION}

We have presented a modified iterative algorithm for the finite element computation of the scattered field by a collection of convex obstacles, based on a reformulation of the problem as coupled single obstacles scattering subproblems. A model order reduction is performed thanks to the introduction of the Macro Basis Functions, to compute an approximate solution within a 


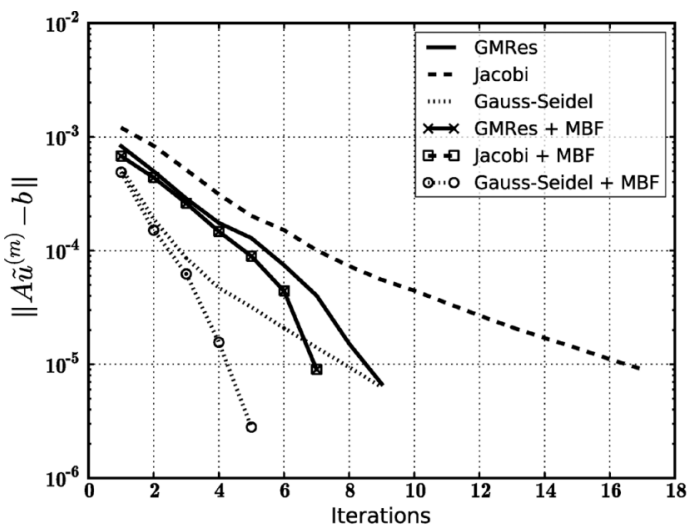

(a)

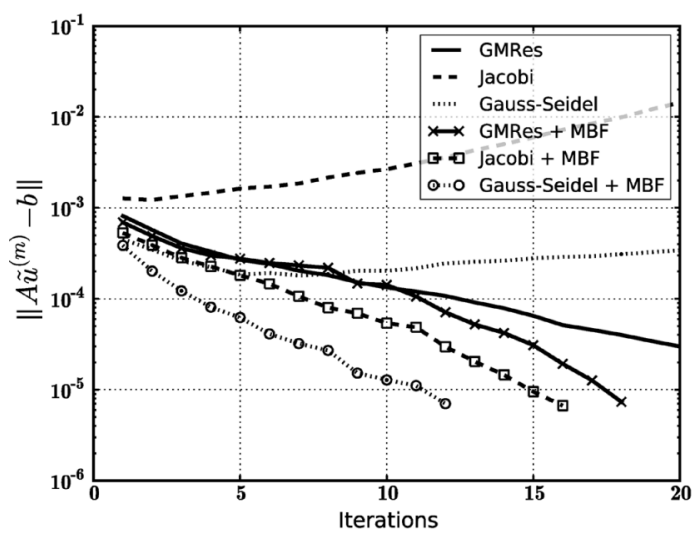

(c)

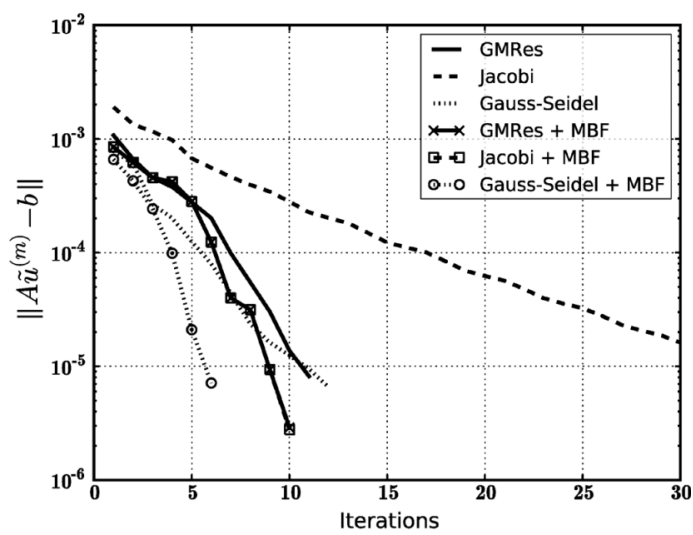

(b)

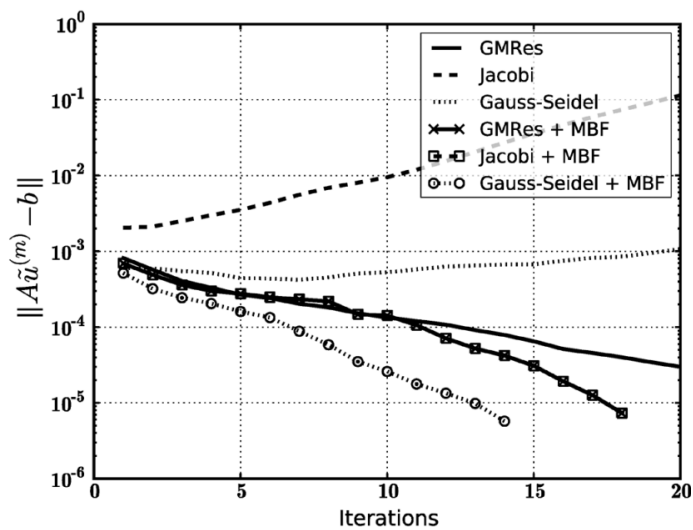

(d)

Fig. 3. Convergence curves for the three different solvers, with and without the model reduction technique to the scattering by the corresponding configurations of obstacles in Fig. 2, with $k R=11$. GMRes: $x_{0}=0$ in all cases except (c), where $x_{0}=b$; Jacobi and Gauss-Seidel: $x_{0}=b$ in all cases.

subspace of solutions. The basis for this subspace is progressively built as the iterative process advances, and is stopped when achieving a residue-based criterion.

The proposed method leads to significantly faster convergence with all three investigated linear solvers, as well as to improved stability. The drawback is an increased memory use. From a convergence point of view, the modified Gauss-Seidel method shows the best performance, although all three modified solvers behave similarly. This contrasts with the original algorithm where significantly different convergence rates were observed, and Krylov subspace methods were usually preferable.

\section{ACKNOWLEDGMENT}

This work was supported in part by the Belgian Science Policy (IAP P6/21), the Walloon Region (Wist 2 616420), and the Belgian French Community (ARC 09/14-02).

\section{REFERENCES}

[1] C. Geuzaine, O. Bruno, and F. Reitich, "On the $\mathcal{O}(1)$ solution of multiple-scattering problems," IEEE Trans. Magn., vol. 41, no. 5, pp. 1488-1491, May 2005.

[2] O. Bruno, C. Geuzaine, J. Monro, Jr., and F. Reitich, "Prescribed error tolerances within fixed computational times for scattering problems of arbitrarily high frequency: The convex case," Philosophical Trans. Royal Soc. (Series A: Math., Phys. Eng. Sci.), vol. 362, no. 1816, pp. 629-645, 2004.
[3] C. Geuzaine, A. Vion, R. Gaignaire, P. Dular, and R. V. Sabariego, "An amplitude finite element formulation for multiple-scattering by a collection of convex obstacles," IEEE Trans. Magn., vol. 46, no. 8, pp. 2963-2966, Aug. 2010.

[4] C. Geuzaine, J. Bedrossian, and X. Antoine, "An amplitude formulation to reduce the pollution error in the finite element solution of time-harmonic scattering problems," IEEE Trans. Magn., vol. 44, no. 6, pp. 782-785, Jun. 2008.

[5] X. Antoine and C. Geuzaine, "Phase reduction models for improving the accuracy of the finite element solution of time-harmonic scattering problems I: General approach and low-order models," J. Comput. Phys., vol. 228, no. 8, pp. 3114-3136, 2009.

[6] X. Radu and C. Craeye, "Fast method to compute an efficient basis to simulate metamaterials with macro basis functions," presented at the 23rd Int. Rev. Progr. Appl. Computational Electromagn., Verona, Italy, 2007.

[7] M. Balabane, "Boundary decomposition for helmholtz and maxwell equations. I. Disjoint sub-scatterers," Asymptot. Anal., vol. 38, no. 1, pp. 1-10, 2004.

[8] J. Yeo, V. V. S. Prakash, and R. Mittra, "Efficient analysis of a class of microstrip antennas using the characteristic basis function method (cbfm)," MOTL, vol. 39, no. 6, pp. 456-464, 2003.

[9] A. Bayliss, M. Gunzburger, and E. Turkel, "Boundary conditions for the numerical solution of elliptic equations in exterior regions," SIAM J. Appl. Math., vol. 42, pp. 430-451, 1982.

[10] Y. Saad, Iterative Methods for Sparse Linear Systems, 2nd ed. Warrendale, PA: SIAM, 2003.

[11] A. Greenbaum, Iterative Methods for Solving Linear Systems. Warrendale, PA: SIAM, 1997.

[12] C. Craeye, "On the connection between multiple-scattering based macro basis functions and Krylov subspace methods," presented at the ICEAA Conf., Torino, Italy, 2009. 